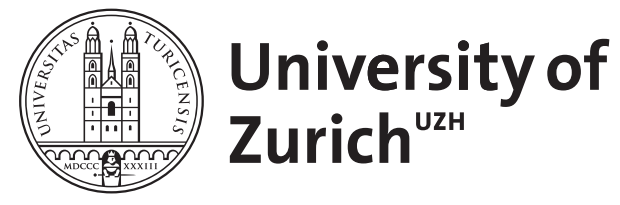

Influence of network properties on a migration induced secular height trend by Monte Carlo simulation

Fritz, Amelie ; Makeyeva, Angelina ; Staub, Kaspar ; Groth, Detlef

DOI: https://doi.org/10.1127/anthranz/2019/1032

Posted at the Zurich Open Repository and Archive, University of Zurich ZORA URL: https://doi.org/10.5167/uzh-172486

Journal Article

Accepted Version

Originally published at:

Fritz, Amelie; Makeyeva, Angelina; Staub, Kaspar; Groth, Detlef (2019). Influence of network properties on a migration induced secular height trend by Monte Carlo simulation. Anthropologischer Anzeiger:1-11. DOI: https://doi.org/10.1127/anthranz/2019/1032 


\title{
Influence of network properties on a migration induced secular height trend by Monte Carlo simulation
}

\author{
Amelie Fritz¹, Angelina Makeyeva², Kaspar Staub³, and Detlef Groth',*
}

\author{
1 University of Potsdam, Institute of Biochemistry and Biology, Bioinformatics Group, Karl-Liebknecht-Str. 24-25, 14476 \\ Potsdam-Golm, Germany \\ 2 Mitteldamm 31a, 14482 Potsdam, Germany \\ 3 Institute of Evolutionary Medicine, University of Zürich, Switzerland \\ * Corresponding author: dgroth @uni-potsdam.de
}

With 3 figures and 5 tables

\begin{abstract}
Background: Recent research reported height biased migration of taller individuals and a Monte Carlo simulation showed that such preferential migration of taller individuals into network hubs can induce a secular trend of height. In the simulation model taller agents in the hubs raise the overall height of all individuals in the network by a community effect. However, it could be seen that the actual network structure influences the strength of this effect. In this paper the background and the influence of the network structure on the strength of the secular trend by migration is investigated. Material and methods: Three principal network types are analyzed: networks derived from street connections in Switzerland, more regular fishing net like networks and randomly generated ones. Our networks have between 10 and 152 nodes and between 20 and 307 edges connecting the nodes. Depending on the network size between 5.000 and 90.000 agents with an average height of $170 \mathrm{~cm}$ (SD $6.5 \mathrm{~cm}$ ) are initially released into the network. In each iteration new agents are regenerated based on the actual average body height of the previous iteration and, to a certain proportion, corrected by body heights in the neighboring nodes. After generating new agents, a certain number of them migrated into neighbor nodes, the model let preferentially taller agents migrate into network hubs. Migration is balanced by back migration of the same number of agents from nodes with high centrality measures to less connected nodes. The latter is random as well, but not biased by the agents height. Furthermore the distribution of agents per node and their correlation to the centrality of the nodes is varied in a systematic manner. After 100 iterations, the secular trend, i.e. the gain in body height for the different networks, is investigated in relation to the network properties. Results: We observe an increase of average agent body height after 100 iterations if height biased migration is enabled. The increase rate depends on the height of the neighboring factor, the population distribution, the relationship between population in the nodes and their centrality as well as on the network topology. Networks with uniform like distributions of the agents in the nodes, uncorrelated associations between node centrality and agent number per node, as well as very heterogeneous networks with very different node centralities lead to biggest gains in average body height. Conclusion: Our simulations show, that height biased migration into network hubs can possibly contribute to the secular trend of height increase in the human population. The strength of this "tall by migration" event depends on the actual properties of the underlying network. There is a possible significance of this mechanism for social networks, when hubs are represented by individuals and edges as their personal relationships. However, the required high number of iterations to achieve significant effects in more natural network structures in our models requires further studies to test the relevance and real effect sizes in real world scenarios.
\end{abstract}

Keywords: secular trend; body height; simulation; community effect; Monte Carlo method; network; centrality measures

\section{Introduction}

The secular trend for the increase in body height of the human population has been intensively studied in recent research. The most prominent example are the Netherlands where the average body height of men has increased by around $20 \mathrm{~cm}$ during the past 150 years (Stulp \& Barrett 2016). However, this tendency is not universal for all coun- tries: for instance, men in the US were the tallest from the end of the $18^{\text {th }}$ until the beginning of the $20^{\text {th }}$ century; thereafter the increase in body height was much smaller than in other populations, only $6 \mathrm{~cm}$ after the beginning of the $20^{\text {th }}$ century (Komlos 2010). The reasons for the difference in body height increase have been discussed intensively. With regards to the Netherlands and the US having similar calorie intake: despite higher per-capita income and more yearly 
investment in health care in the US, the secular trend was much higher in the Netherlands. Moreover, for black US American women even a decrease in average body height was reported for birth cohorts from 1975 to 1986 (Komlos 2010). Similarly, there was also a lack of a secular trend for South African military conscripts between 1946 and 1995 whereas in Swiss and Dutch conscripts it was (Myburgh et al. 2017). The reasons for this might be due to socioeconomic or social effects caused by increased inequalities in the US or in South Africa. In contrast, the increase of percapita income, which was recently claimed to be associated with an increase in average body height (Steckel 1983; Baten $\&$ Blum 2012), could not offer a sufficient explanation of the different developments in those countries.

In addition to explanations of the secular trend, mostly of socioeconomic nature, e.g. health and nutrition issues (Silventoinen 2003; Steckel 2009), social network effects have been discussed (Aßmann \& Hermanussen 2013). Height adjustments on an individual level within a community, e.g.in kindergarten (Pospisil et al. 2017) and school groups (Koziel \& Gomula 2017), have been observed. Within such small communities variances in body height are much smaller in comparison to the overall population. On a larger network scale, investigations of distributions of average body heights for conscripts in Switzerland (Hermanussen et al. 2014), Poland (Gomula et al. 2017) and Norway (Bents et al. 2017) showed height clustering towards the average height. People living in districts, cities connected by road connections, have similar body heights.

Such neighbor body height correlations and network differences between various regions, can be generated by a simple Monte Carlo simulation as shown recently by Hermanussen et al. (2016). Using the road network of Switzerland, similar body height clustering patterns as well as varying shifts between "tall" and "short" areas have been observed when a neighboring factor of around $5-10 \%$ was introduced. In this simulation body height of the next generation agents was based to $90-95 \%$ on the height of the actual node and to $5-10 \%$ on the height of the nodes directly connected to this node. This approach resulted in real world spatial height distributions even if the model was started with a homogeneous network. However, this simulation could not explain the secular trend; the average body height remained unchanged during the simulation. Based on the observation that stature often represents status, i.e. occupation of social network hubs by taller individuals, and that migrants seem to be taller than non-migrants (Kobyliansky \& Arensburg 1977; Singh \& Harrison 1996; Krzyzanowska \& Mascie-Taylor 2011), the model developed by Groth (2017) was extended by introducing a height biased migration where taller individuals preferentially migrated into network hubs. This approach resulted, due to the neighboring factor, the community effect, in overall gain of body height of the whole system. However, there were large differences in body height gains between different networks; the reasons for this remained unclear.
In this paper we investigate the effect of network structure on the effect size of the secular trend by height biased migration. We evaluate different network structures based either on real world street networks of Switzerland, homogeneous fishing net like networks and different random networks. Basic network properties as well as different distributions of the agent population in a network are used to evaluate the reasons for different body height gains in different networks and societies.

\section{Methods}

We use a network based approach for our simulation where we build different network / graphs to perform our analysis. A graph consists of nodes and edges connecting those nodes. We can think in real world terms of nodes as cities with people living there and edges as streets connecting those cities. For studying the influence of network topology and network properties on the secular trend induced by migration, we investigate five different graph types in different sizes. The chosen graph size classes are graphs with up to 20 nodes (mini), between 21 and 35 nodes (midi) and with up to 152 nodes. In our simulations we use random graphs based on standard graph generation algorithms, such as the Erdös-Renyi graphs (erren, emidi) (Erdös \& Renyi 1959), scale-free graphs (bmidi) according to the BarabasiAlbert model (Barabasi \& Albert 1999), small world graphs (wmidi) (Watts \& Strogatz 1998), and a self-implemented graph algorithm (amini, amidi, angie).

The latter networks are generated as follows: After initially adding edges at random between nodes, not yet connected nodes are connected, in the second phase, after all nodes are at least connected by one edge, we use random edge additions. In the last step, after all edges are added to the graph, nodes in separate graph components are preferentially connected by random switching of "within components edges" to "edges between components". The process is finished when all nodes are within the same component.

Furthermore, fishing net like graphs are constructed with various centralization additions, fmidi and fnet. Three levels of centralization are implemented in the fishing net like graph: 1 is the lowest and 3 is the highest. These levels are achieved by adding more edges to centralize some nodes in this network type.

We also investigate graph types derived from the geography of Switzerland smini, smidi, swiss, swiso (Hermanussen et al. 2014; Hermanussen et al. 2016; Groth 2017). The mini and midi forms of this network type are extracted communities within the Switzerland network graph. Communities are densely connected nodes within a graph. In the swiso graph we take the original number of conscripts per district in 2005 to populate the graph with agents, whereas in all the other graphs we use a log-normal distribution to determine the node population. Some of the used network topologies are 
shown in Fig. 1, basic network properties of the generated graphs are summarized in Table 1.

After graph creation, agents with an average body height of $170 \mathrm{~cm}(\mathrm{SD}=6.5)$ are added to the nodes. With the exception of the swiso network, where the number of agents we use is drawn from the conscript data from 2005, all other networks receive a number of agents per node which is randomly attributed by a log-normal distribution (meanlog $=6$, sdlog varied between 0.01 and 1 ). The setting with a meanlog of 6 and a sdlog of 0.6 or 0.8 is closest to the distribution of conscript numbers in Switzerland in 2005 in the swiso graph. As the correlation between node centrality and number of agents per node might vary considerably between different networks, we also adapt the assignment of agent numbers to each node by adjusting for the final Pearson correlation coefficient ( $\mathrm{r}$ ) for the association between node degree and agent number. We vary $r$ between 0.0 and 0.6 in steps of 0.15 . It should be noted, that in the original swiso network the correlation between the number of agents taken from 2005 and the node degree based on the Switzerland road-map was 0.447. This value is within the range of our experimental variations.

After distributing the agents into the networks Monte Carlo simulations are started by generating subsequent generations based on the 1) actual node body height mean,

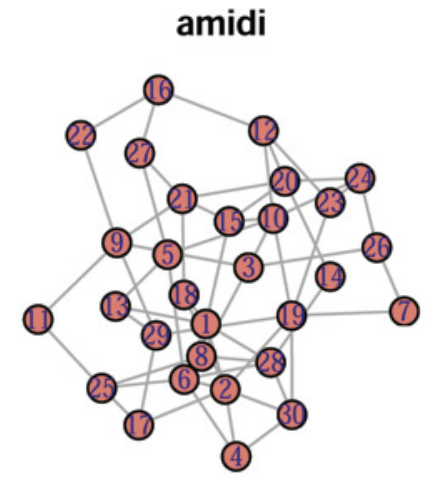

fmid1

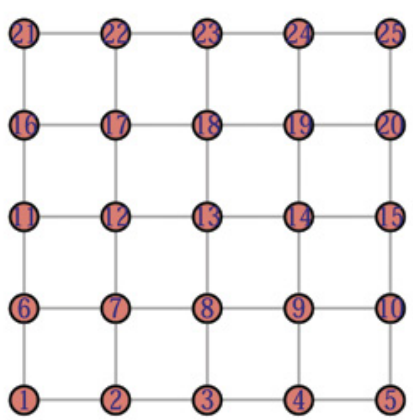

smidi

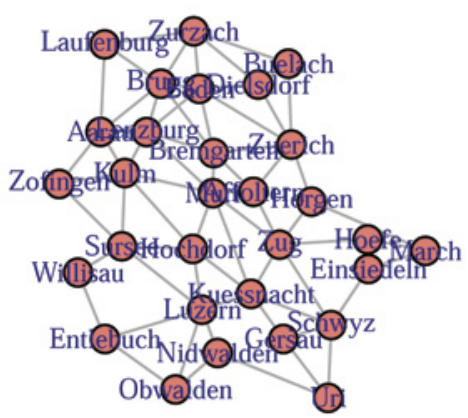

bmidi

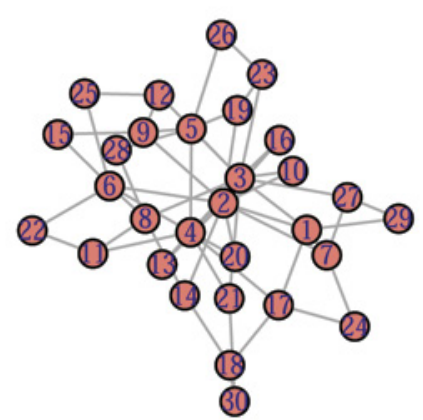

fmid2

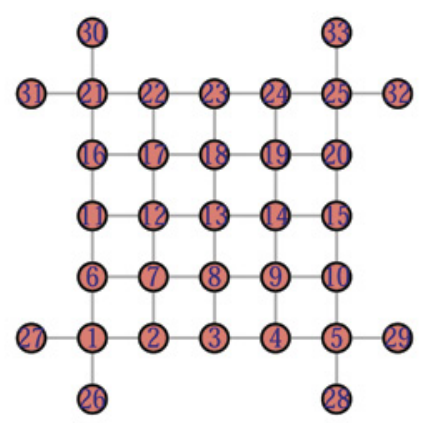

swiso

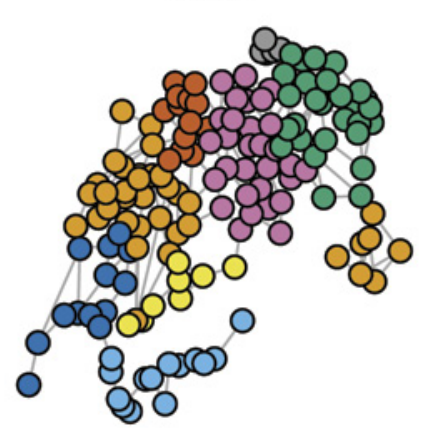

emidi

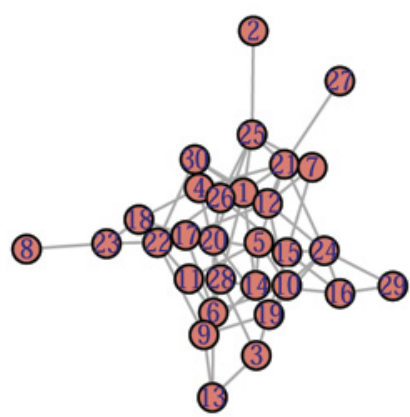

fmid3

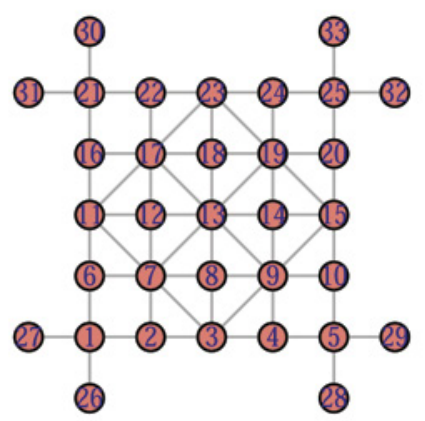

swrnd

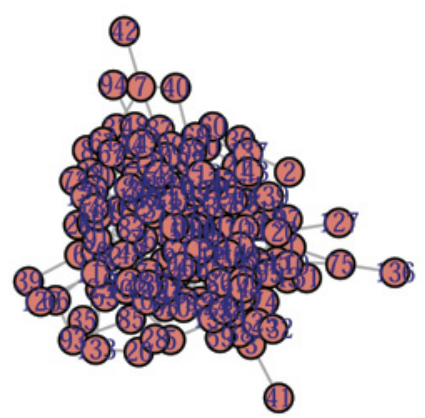

Fig. 1. Sample network topologies. Medium sized graphs with 30-36 nodes based on random graph algorithms are shown in the first row. The second row contains regular fishing net like graphs and the third row contains graphs based on the topology of the Switzerland network. The smidi graph is a medium sized component of the Switzerland map, shown on the left hand side. The swrnd is a randomized version of the swiso graph where edges were randomly reassigned while retaining the same degree distribution as in the swiso network. 
Table 1. Graph properties. v: number of vertices, e: number of edges, dia: graph diameter, agents: number of agents with sdlog = 0.8 , $\mathrm{m}$ : node means of centrality measures for degree, betweenness and page rank values, cv.deg: coefficient of variation for node degrees, gi.deg Gini index for node degrees, as.deg: assortativity values for node degrees, tr.gl: global transitivity. In case of random graphs, the averages for five random graphs are shown.

\begin{tabular}{|l|c|c|c|c|c|c|c|c|c|c|c|}
\hline & $\mathbf{v}$ & e & dia & agents & m.deg & m.bet & m.prk & cv.deg & gi.deg & as.deg & tr.gl \\
\hline amini & 10 & 20 & 3 & 5648 & 4.0 & 2.9 & 0.100 & 27.9 & 0.15 & -0.18 & 0.38 \\
\hline amidi & 30 & 60 & 5 & 17850 & 4.0 & 21.9 & 0.033 & 37.9 & 0.21 & -0.02 & 0.12 \\
\hline angie & 144 & 300 & 7.8 & 75636 & 4.2 & 191.0 & 0.007 & 41.9 & 0.23 & 0.00 & 0.03 \\
\hline bmidi & 30 & 57 & 4.6 & 13908 & 3.8 & 20.9 & 0.033 & 65.8 & 0.32 & -0.20 & 0.16 \\
\hline emidi & 30 & 62 & 5.6 & 15640 & 4.1 & 21.9 & 0.033 & 45.9 & 0.26 & -0.06 & 0.13 \\
\hline erren & 148 & 295 & 8 & 83386 & 4.0 & 203.8 & 0.007 & 46.4 & 0.26 & 0.04 & 0.03 \\
\hline fmid1 & 25 & 40 & 8 & 11125 & 3.2 & 28.0 & 0.040 & 22.1 & 0.12 & 0.42 & 0.00 \\
\hline fmid2 & 33 & 48 & 10 & 21727 & 2.9 & 50.2 & 0.030 & 40.6 & 0.21 & -0.30 & 0.00 \\
\hline fmid3 & 33 & 60 & 8 & 18934 & 3.6 & 38.3 & 0.030 & 55.8 & 0.31 & 0.04 & 0.32 \\
\hline fnet1 & 144 & 264 & 22 & 79121 & 3.7 & 500.5 & 0.007 & 14.4 & 0.07 & 0.59 & 0.00 \\
\hline fnet2 & 152 & 272 & 24 & 80002 & 3.6 & 560.0 & 0.007 & 21.0 & 0.09 & 0.22 & 0.00 \\
\hline fnet3 & 152 & 290 & 19 & 83364 & 3.8 & 488.6 & 0.007 & 29.5 & 0.14 & 0.21 & 0.12 \\
\hline smini & 13.8 & 19 & 7.4 & 8924 & 2.7 & 13.7 & 0.073 & 37.3 & 0.20 & -0.09 & 0.30 \\
\hline smidi & 29 & 63 & 6.2 & 15475 & 4.3 & 27.3 & 0.035 & 34.2 & 0.19 & 0.19 & 0.39 \\
\hline swiso & 148 & 307 & 30 & 81576 & 4.1 & 564.6 & 0.007 & 40.8 & 0.23 & 0.36 & 0.36 \\
\hline swrnd & 148 & 307 & 7.2 & 83435 & 4.1 & 197.3 & 0.007 & 40.8 & 0.23 & -0.01 & 0.03 \\
\hline wmidi & 36 & 72 & 5.4 & 18534 & 4.0 & 29.7 & 0.028 & 26.4 & 0.14 & -0.02 & 0.08 \\
\hline
\end{tabular}

2) neighbor node influence of different strengths $(0,0.05,0.1$, 0.2 and 0.5 ) to simulate the community effect in height and 3) by height biased migration where taller individuals tend to migrate preferentially into network hubs. Three centrality measures were used to determine the direction of migration: the node degree measure as the number of edges adjacent to one node, the betweenness centrality as the number of shortest paths between all nodes traversing the node, and the pagerank centrality which is based not only on the nodes own centrality but as well take into consideration the centrality of nodes, closely connected to the node. Contrasting to Groth (2017) we do not use the square root of the number of agents in the node to determine the number of migrating agents, but let $10 \%$ of the population of the node with fewer agents migrate. That way we avoid possible bias due to very different network sizes used in this study. We sample randomly $10 \%$ of agents from the less central node and from the taller half of its population to migrate towards more central nodes. Back migration is done with the same number of agents, but is independent from height. More background information of this Monte Carlo simulation can be found in Groth (2017).

\section{Statistical methods}

Analysis and modeling is performed using the statistical programming language $R$ ( $R$ Core Team 2018) with the stan- dard $\mathrm{R}$ packages and the additional packages igraph (Csardi \& Nepusz 2006), DescTools (Signorell et al. 2018), e1071 (Meyer et al. 2017), xtable (Dahl 2016), corrplot (Wei \& Simko 2017), mapdata (Becker et al. 2016), geosphere (Hijmans 2017), sp (Pebesma \& Bivand 2005, Bivand et al. 2013) and optparse (Davis 2018). Data for the administrative map of Switzerland was taken from the website https://www. gadm.org (Hijmans et al. 2016).

The dependencies of changes in body height in the networks in relation to the network properties are analyzed using a multiple linear regression model. Statistical significance of differences between network types is evaluated using analysis of variance model or, in case of pairwise comparisons, using a two-sample t-test. In case of ANOVA pairwise t-tests are performed in post-hoc analysis using p-value corrections by Holm (Holm 1979). Pearson correlations are used to describe spatial correlations between neighboring nodes or autocorrelations for the same node at different time points. In order to compare different distributions two-sample Kolmogorov-Smirnov tests are performed (Kolmogorov 1933; Smirnov 1939). In case of averages, or correlation $95 \%$ coefficients confidence intervals are given in rectangular braces. For all tests standard functions of the R stats package are used (R Core Team 2018).

At least five different Monte Carlo simulations are performed for each graph type generated by random algorithms. Final model evaluation is done after 100 iterations. 


\section{Results}

\subsection{Population distribution}

Preliminary analyses reveal that the distribution for the numbers of agents per districts might have a large influence on the model. We therefore try several distributions, more or less similar to the number of conscripts per district in the original Switzerland model. We find that the number of agents per district follow a log-normal distribution with a meanlog of 6.00 and a sdlog of 0.85 , the one-sample Kolmogorov-Smirnov test for this distribution and the agent distribution in the Switzerland graph swiso is not significant for this setting, $p=0.18$. Therefore we assume that such settings for the population are very similar to the Switzerland network.

As the distribution of the population may not follow real world scenarios like that of the swiso graph, we decided to use sdlog values of $0.01,0.2,0.4,0.6,0.8,1.0$. We cannot use 0.00 , because we also change the correlation between the degree centrality and the number of agents of a node. Therefore we need at least a small difference in the number of agents to construct a sequence of correlation coefficients. The correlation between the degree of a node and the number of agents in the original swiso graph is $\mathrm{r}=0.45, \mathrm{CI} 95 \%$ $=[0.31,0.57]$. To analyze the effect of correlation between the node centrality and the number of agents we modify the association between both variables in steps of 0.15 from 0.0 to 0.6 .

As in Groth (2017) we investigate the influence of the neighbor factor on our migration model using influence proportions of 0 (null model, no influence of neighbors), to 0.05 , $0.1,0.2$ and 0.5 , the latter represents a $50 \%$ influence from the neighbors on the final body height of the node. Thereby, at each iteration the height of the node agents is calculated to $50 \%$ from the height of the neighbors and to $50 \%$ from the current note height value. Those values are slightly adjusted to take the number of agents per node into consideration. For details see Hermanussen et al. (2016).

Figure 2 summarizes the influence of the population distribution and neighbor factor on the final body height for the swiss graph. Result pattern for the pattern of increase are similar in other graph types, however, the maximal final body height after 100 iterations is different between the graph types (see section 4.2). In all graph types, in case of medium and low correlations $(\mathrm{r} \leq 0.3)$ between node centrality and number of agents per node an increase in body height with increasing neighbor factor is observed. In experiments with higher correlations between node centrality and number of agents per node, meaning that the networks have highly connected nodes with much more inhabitants than their neighboring nodes (megacity nodes), this tendency is smaller or even reversed, as can be seen in the panel for $\mathrm{r}=$ 0.6 and $\operatorname{sdlog}=1$.
To investigate this tendency in more detail, a linear regression to determine the effect of those variables on body height is used. Table 2 summarizes the results for the three centrality measures, degree, betweenness and page rank centrality. For all measures we find a significant positive influence of the neighbor factor and the agent migration; on the other hand a significant negative effect of the increasing associations between the node degree centrality and the number of agents per node as well as a negative influence of unequal distribution of the agents into the nodes is observed. This means that if there are only a few centralized nodes with much more agents than their neighbors, the overall increase body height in the network is smaller. The same negative trend is observed if the sdlog is raised. Body height increase is largest in situations where there is an almost homogeneous distribution of agents into the nodes, i.e. the number of agents per node follows a homogeneous distribution.

\subsection{Network topology and size}

In our further analyses we focus on settings close to the natural swiss network using a correlation coefficient of 0.45 for the association of degree centrality and number of agents per node, a sdlog of 0.6 for the population distribution and a neighbor factor of 0.2 . With those settings we investigate the effect of network structure and network size on the average body height in the network after 100 iterations if height biased agent migration mediated by degree centrality is enabled (Fig. 3).

We observe that average body height of agents is positively associated with network heterogeneity. When the node degrees of the network had a very large variation, the final body height of the agents is greater in comparison to more homogeneous networks with nodes having more similar node degrees $\left(\mathrm{R}^{2}=0.55\right.$, see Table 3$)$. Not surprisingly, this is reflected, although with a lower $\mathrm{R}^{2}$ increase, by the number of migratory roads in the network. Migratory roads are edges between two nodes where the centrality measures between the nodes vary. For instance, between two nodes with the same degree there is no height biased migration, whereas between nodes with different centrality measures there is.

There is further a much smaller influence of the network size, reflected by the number of nodes and the total number of agents in the network, on final body height. Obviously, if there are more agents in the network while the number of nodes remains constant, the number of migrants with the same migrant proportion (10\% from the taller half) should increase. However, the balance between small and large nodes might be more shifted to nodes with more agents, therefore migration could be also reduced. The increase in population in a log-normal distribution might be dominated by the agent numbers in the few highly populated nodes. Summarizing the individual coefficients for the network: the 


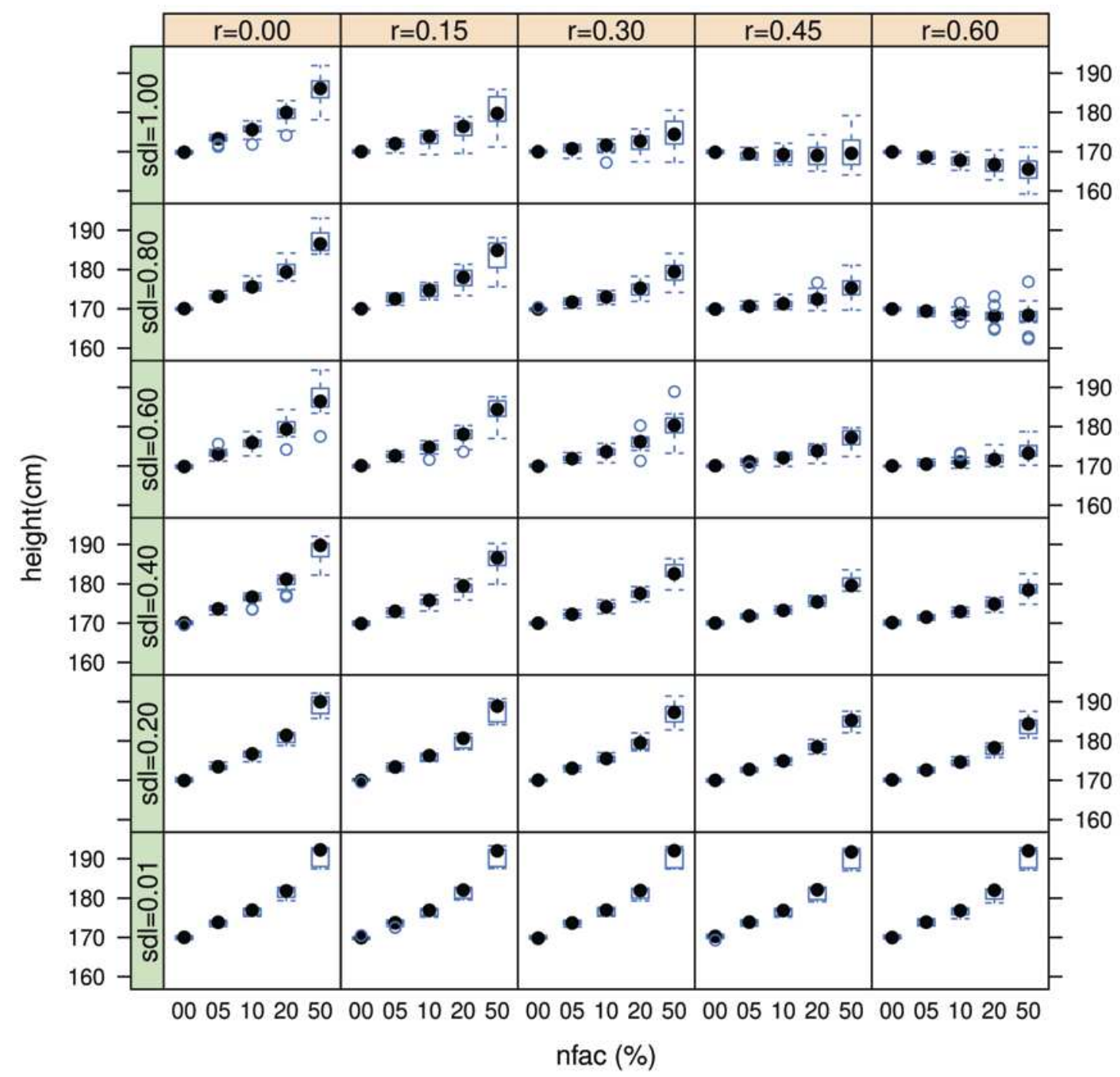

Fig. 2. Influence of neighbor factor (nfac), centrality to population correlation ( $r$ ) and population distribution (sdlog, 0.01 very even, uniform, 1.0 log-normal) on body height for the swiss graph after 100 iterations using boxplots.

positive association of body height increase to network heterogeneity (cv.deg, mig.prop) and the vertex numbers can be confirmed. In contrast the number of agents per network is negatively associated to the final average body height (Table 4).

Finally we compare different networks of medium size concerning their responsiveness to a migratory effect on body height. In agreement to our linear model for all networks together, in networks with higher levels of heterogeneity the final average agent body height is larger (Table 5), e.g. the scale-free Barabasi-Albert network-bmidi, has the highest variation in the degree distribution and the highest gain in body height. In contrast the networks with the lowest variation in degrees, that small-world network wmidi and the sub-network of Switzerland smidi are also the networks with the lowest gain in body height.

\section{Discussion}

The enormous increase in human body height during the last two centuries has drawn a lot of attention in the scientific community (Hatton \& Bray 2010, NCD Risk Factor Collaboration 2016). Explanations for this phenomenon usually include effects due to nutritional, medical and environmental improvements (Silventoinen 2003; Steckel 2009). The discussion of genetic sources has produced controversial conclusions (Rietveld et al. 2004; Stulp et al. 2012; Stulp \& Barrett 2016; Stulp et al. 2017). But in the short period of time genetic variations should have a low impact on body height. As humans are social beings and personal self-perception is of great importance, community effects on body height: "As tall as my peers ..." (Bogin et al. 2018) might influence body height and therefore contribute to the secu- 
Table 2. Linear modeling of body height for the swiss network for different centrality measures. The independent variables are: nfac neighborhood factor, the higher nfac the higher the neighborhood influence, mig - migration, 1 one for migration, 0 for no migration, r.tar - target correlation between degree of nodes and number of agents per node, with higher r.tar the number of megacity nodes increases, sdlog - generation of population distribution, the higher the sdlog the more uneven the distribution of the agent numbers per node. All factors are significant for the model $(p<0.05)$. The values for r.squared in the last column are for the complete model.

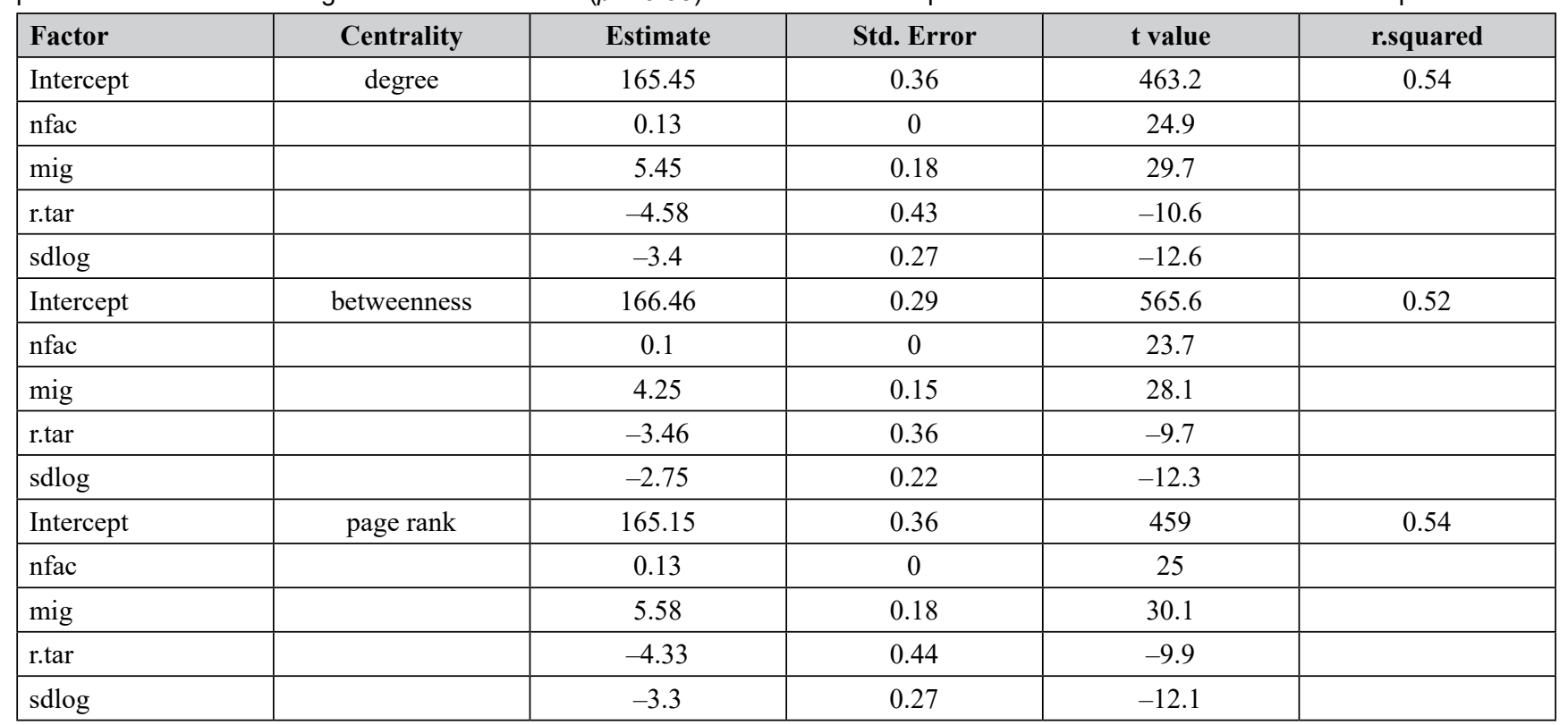

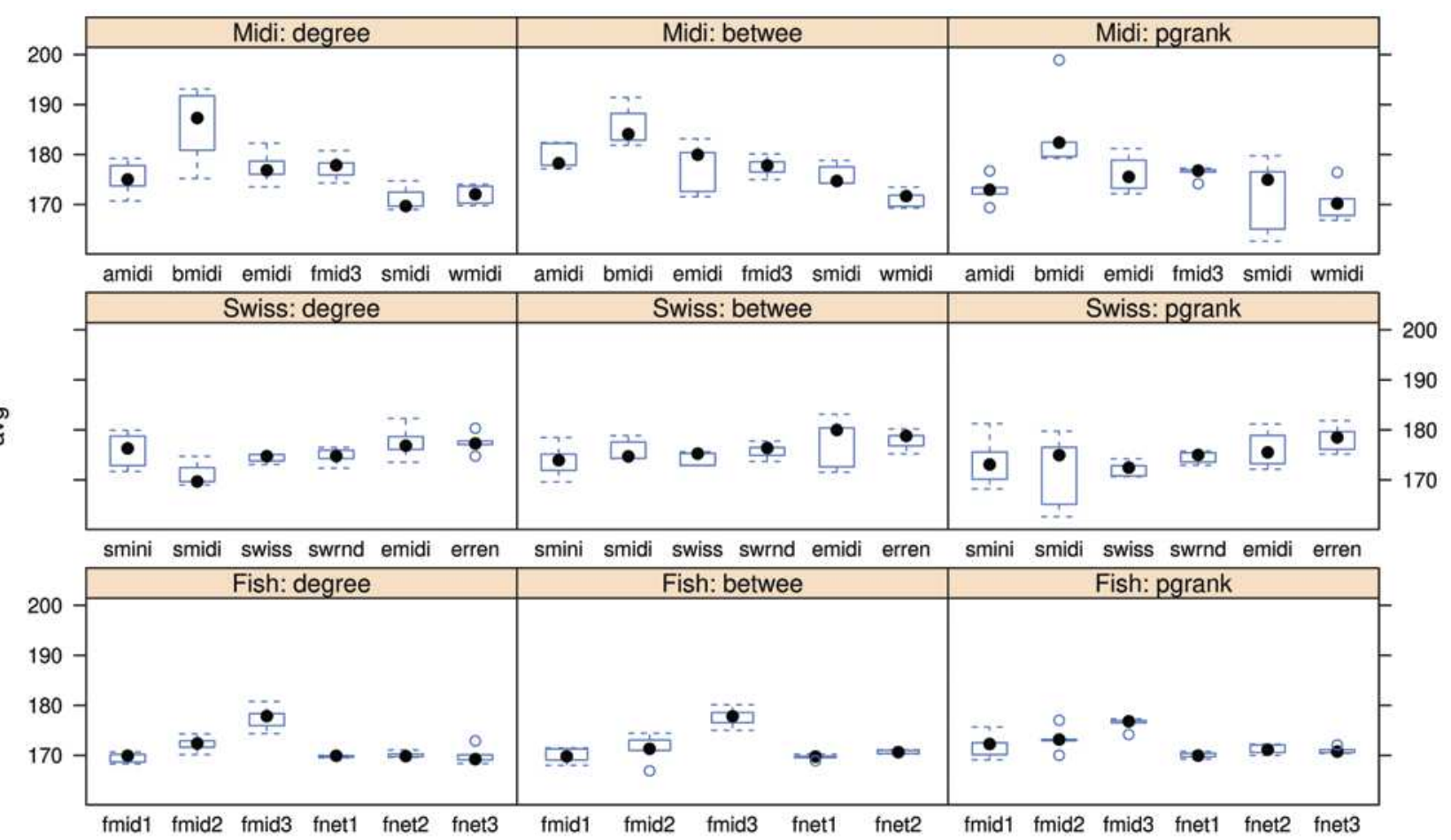

Fig. 3. Influence of the network structure on body height increase induced by migration in the fishnet like networks, bottom figures, swiss like and random network, middle row figures and medium sized networks based on different network topologies. In the left column migration is based on node degree centralities, middle column on betweenness centralities and in the right column on page rank centralities. Settings for the analysis were neighbor factor of 0.2 , sdlog of 0.6 and r.tar of 0.45 . 
Table 3. Linear model after forward selection for investigating the effect of network structure on final body height of agents after 100 iterations. Abbreviations used are: avg - average body height after 100 iterations, cv.deg - coefficient of variation of node degrees, people - number of agents per network, nvertex - number of vertices per network, mig.prop - proportion of migratory roads in the network. Settings for this analysis are nfac of 0.2 , r.tar of 0.45 and sdlog of 0.6 , and node degrees to determine direction of migration.

\begin{tabular}{|l|c|c|c|}
\hline Formula & R.squared & AIC & $p$-value \\
\hline avg $\sim$ cv.deg & 0.55 & 173.9 & $<0.001$ \\
\hline avg $\sim$ cv.deg + people & 0.62 & 171.1 & $<0.001$ \\
\hline avg $\sim$ cv.deg + people + nvertex & 0.67 & 168.5 & $<0.001$ \\
\hline avg $\sim$ cv.deg + people + nvertex + mig.prop & 0.7 & 168.3 & $<0.001$ \\
\hline
\end{tabular}

Table 4. Coefficients for the variables of the final linear model chosen by forward selection where initially the variable with the highest $\mathrm{R}^{2}$ value is taken into the model and thereafter stepwise other variables which improve the model considerably. Settings for this analysis are nfac of 0.2 , r.tar of 0.45 , sdlog of 0.6 and using node degrees to determine direction of migration. Abbreviations used are: cv.deg - coefficient of variation of node degrees, people - number of agents per network, nvertex - number of vertices per network, mig.prop - proportion of migratory roads in the network.

\begin{tabular}{|l|c|c|c|c|}
\hline & Estimate & Std. Error & t value & Pr $(>|\mathbf{t}|)$ \\
\hline (Intercept) & 140.03 & 12.19 & 11.49 & 0.00 \\
\hline cv.deg & 0.35 & 0.08 & 4.40 & 0.00 \\
\hline people & -0.70 & 0.34 & -2.05 & 0.05 \\
\hline nvertex & 0.67 & 0.37 & 1.82 & 0.08 \\
\hline mig.prop & 13.56 & 9.88 & 1.37 & 0.18 \\
\hline
\end{tabular}

Table 5. Relationship between final body height and network heterogeneity. avg: average agent body height after 100 iterations for at least five Monte Carlo simulations, ci.low and ci.up borders of the 95 percent confidence interval, cv.deg average coefficient of variation of the random generated 5 networks. Settings for this analysis are nfac of 0.2 , r.tar of 0.45 , sdlog of 0.6 and using node degrees to determine direction of migration.

\begin{tabular}{|l|c|c|c|c|}
\hline network & avg & ci.low & ci.up & cv.deg \\
\hline bmidi & 185.6 & 176.3 & 195 & 65.8 \\
\hline emidi & 177.5 & 173.5 & 181.5 & 45.9 \\
\hline fmid3 & 177.4 & 174.4 & 180.5 & 55.8 \\
\hline amidi & 175.3 & 171.1 & 179.5 & 37.9 \\
\hline wmidi & 172 & 169.6 & 174.4 & 26.4 \\
\hline smidi & 171.1 & 168.1 & 174.1 & 34.2 \\
\hline
\end{tabular}

lar trend. A study on Meerkat, where competing growth to achieve social dominance took place (Huchard et al. 2016), and the observation that network hubs are preferentially occupied by tall people (Stulp et al. 2013; Hermanussen \& Scheffler 2016) inspired us to perform this study.

Therefore we investigated in more detail the possible effect of migration on the secular trend if migration is biased on height. Studies suggest that migrants generally might be taller than residential persons (Singh \& Harrison 1996). In agreement with this observation are for instance studies from the UK (Krzyzanowska \& Mascie-Taylor 2011). Furthermore, in the $19^{\text {th }}$ century, people in the region with the largest number of immigrants, North America, were the tallest in the world (Komlos 2009). In addition it is important to note, that the secular trend started around 150 years ago, when the possibilities for mobility and migration were greatly improved due to human achievements in traffic and transport using railways, cars and airplanes (Hatton 2013). Hatton (2013) explains the gain in body height by easier access to high quality food resources due to the traffic improvements. Grasgruber et al. (2016) also analyzed food quality and quantity as the major determinants of human height. In principle, mobility and gain in body height are positively associated likewise the other socioeconomic factors changing in the past. We postulate that not only the improved food supply through the expansion of the transport infrastructure, 
but also the strengthening of the community connections has an influence on the body height of the population. Mobility might lead to height gain. For instance comparing the average body height of settled and nomadic Turkana of Kenya, Little et al. (1993) found that nomadic Turkana were 1-2 cm taller than their settling counterparts. Heterosis resulting from the increase in genetic variability due to migration in the urban regions has been proposed too as explanation for the secular trend (reviewed in Bogin 1999).

The fact that a secular trend by migration can exist if the people can adapt their body height to a certain degree to the height of their peers, was recently demonstrated by Groth (2017). However, the increase in agent height varied considerably between the network types. In this study we investigated the influence of population distribution and network topology on a possible secular trend induced by height biased migration of taller individuals.

Our first observation was that the influence of the distribution of the population into the network has a more important role in determining the strength of the secular trend than the network topology or network size. The gain in body height is largest in networks with a more homogeneous distribution of the population in the network. Such distribution was achieved by using low sdlog values for the log-normal function, lower than in our Switzerland reference, such as $0.01,0.2$ or 0.4 . In contrast to this, the existence of highly connected mega cities weakens the effect of migration on height. Settings with a more log-normal distribution of the population and with a high correlation between population of the nodes and their number of connections to other nodes could even reverse the effect of migration on body height. In this case the mega nodes attract tall agents/individuals from the surrounding nodes. However, the average body height is only marginally influenced by in-migration of a few slightly taller agents into the mega city nodes. Opposing to the situation in those central nodes, in the surrounding smaller nodes, the loss of a significant number of tall people decreases the average body height here much more than the increase in the mega city/mega node could compensate. In the neighboring region of the mega node the average body height decreased due to the loss in out-migrating tall agents. In response to the neighbor factor, simulating the community effect, in the adaptation phase of the model, the surrounding nodes diminish the average body height in the mega node more than it gets increased by the previous migration gain. This situation could be observed when the correlation between the degree centrality and the number of agents per node (r.tar) and the sdlog for the log-normal distribution was higher than in the natural reference example in Switzerland. Such settings were for example an sdlog of 1.0 and an r.tar of 0.6. In Switzerland we observed a log-normal distribution with an sdlog between 0.6 and 0.85 and a correlation between node degree and population of 0.45 . If the correlation and the sdlog was lower than in the Switzerland network (sdlog $<0.6, \mathrm{r}<0.45$ ) than the increase in final in body height was much larger for the model.

In the second part of our analysis we investigated the effect of network topology and network size on the "tall by migration" effect. We observed that network size was not important and that the simulation could be done also with smaller networks which are computationally more feasible. We therefore analyzed in more detail different network topologies with node numbers between 25 and 36 and with 40 to 72 edges. The factor, which explained most of the variation, was the coefficient of variation for the node degrees. The higher the inequality in node degrees, the more pronounced the gain in body height. Around 55\% of variation was contributed by this factor alone. Adding the next three important variables, agents per network, number of vertices and amount of migratory roads, only increased the r-squared value by another $15 \%$. As the latter of those three factors should be directly connected to network heterogeneity as well, this underlines the importance of having highly asymmetric network structures also.

In conclusion, the effect of migration on body height increase in our model is largest in situations with a homogeneous distribution of the population and a highly heterogeneous network topology. Nodes with only a few agents in central positions and many connections to the surrounding area trigger, due to their high responsiveness to immigration, are the strongest gain in body height for the whole network. In contrast, highly connected mega nodes with much more agents than nodes in their connected neighbors, buffer or even reverse this effect. It should be mentioned here that the body height gain in networks based on the natural geography of Switzerland has been one of the lowest, only surpassed by the model height increase in the regular fishing net like networks without any hub nodes.

The importance and reliability of those findings for real world situations remains to be questioned. The Swiss type networks for instance responded only marginally on this effect induced by migration. Such effects may be important in social networks where taller individuals might be more present in hubs than in marginal positions (Stulp et al. 2013). We hope that the published observations in this article might convince the reader that a secular trend mediated through "tall by migration" could be a plausible scenario in certain situations of human history.

Acknowledgements: Statistical analyses of data were conducted during the International Summer School "Child and Adolescent Growth and Nutrition" in Potsdam/Gülpe from the $1^{\text {st }}$ to $7^{\text {th }}$ July in 2018. The Summer School was supported by the Auxological Society (Gesellschaft für Auxologie), and by the Society of Anthropology (Gesellschaft für Anthropologie). 


\section{Abbreviations}

amidi

amini

angie

bmidi

emidi

erren

fmidi

fnet

smidi

smini

swiso

swiss

wmidi

medium sized random graph

small random graph

larger random graph

medium sized graph based on the Barabasi-

Albert model

medium sized Erdös-Renyi graph

larger Erdös-Renyi graph

medium sized fishing net like graph, numbers at

the end indicate level of centralization

larger fishing net like graph, numbers at the end indicate level of centralization

medium sized sub-network of the swiss network small sub-network of the swiss network original graph based on the geography of Switzerland and the conscripts numbers in 2005 original graph based on the geography of Switzerland but with random population medium sized small world graph

\section{References}

Aßmann, C., \& Hermanussen, M. (2013). Modeling determinants of growth: Evidence for a community-based target in height? Pediatric Research, 74(1), 88-95. https://doi.org/10.1038/ pr.2013.50 PMID:23493166

Barabasi, A.-L., \& Albert, R. (1999). Emergence of scaling in random networks. Science, 286(5439), 509-512. https://doi.org/ 10.1126/science.286.5439.509 PMID: 10521342

Baten, J., \& Blum, M. (2012). Growing tall but unequal: New findings and new background evidence on anthropometric welfare in 156 countries, 1810-1989. Economic History of Developing Regions, 27(sup1, supl), S66-S85. https://doi.org/10.1080/2078 0389.2012.657489

Becker, R. A., Wilks, A. R. \& Brownrigg, R. (2016). mapdata: Extra Map Databases. $R$ package version 2.2-6. $S$ code by Becker and Wilks. R code by Brownrigg. https://CRAN.R-project. org/package $=$ mapdata

Bents, D., Rybak, A., \& Groth, D. (2017). Spatial conscript body height correlation of Norwegian districts in the $19^{\text {th }}$ century. Anthropologischer Anzeiger, 74(1), 65-69. https://doi.org/ 10.1127/anthranz/2017/0700 PMID:28375426

Bivand, R. S., Pebesma, E., \& Gómez-Rubio, V. (2013). Applied spatial data analysis with $R\left(2^{\text {nd }}\right.$ ed.). NY: Springer. https://doi. org/10.1007/978-1-4614-7618-4

Bogin, B. (1999). Patterns of human growth (Vol. 23). Cambridge University Press.

Bogin, B., Hermanussen, M., \& Scheffler, C. (2018). As tall as my peers - similarity in body height between migrants and hosts. Anthropologischer Anzeiger, 74(5), 365-376. https://doi. org/10.1127/anthranz/2018/0828 PMID:29328347

Csardi, G., \& Nepusz, T. (2006). The igraph software package for complex network research. InterJournal. Complex Systems, 1695. http://igraph.org

Dahl, D. (2016). xtable: Export Tables to LaTeX or HTML. R package version 1.8-2. https://CRAN.R-project.org/package=xtable
Davis, T. (2018). optparse: Command Line Option Parser. R package version 1.6.0. https://CRAN.R-project.org/package $=$ optparse

Erdös, P., \& Renyi, A. (1959). On random graphs. Mathematicae, 6, 290-297.

Gomula, A., Koziel, S., Groth, D., \& Bielicki, T. (2017). The effect of neighboring districts on body height of Polish conscripts. Anthropologischer Anzeiger, 74(1), 71-76. https://doi.org/ 10.1127/anthranz/2017/0701 PMID:28362025

Grasgruber, P., Sebera, M., Hrazdíra, E., Cacek, J., \& Kalina, T. (2016). Major correlates of male height: A study of 105 countries. Economics and Human Biology, 21, 172-195. https://doi. org/10.1016/j.ehb.2016.01.005 PMID:26948573

Groth, D. (2017). Modeling a secular trend by Monte Carlo simulation of height biased migration in a spatial network. Anthropologischer Anzeiger, 74(1), 81-88. https://doi.org/ 10.1127/anthranz/2017/0703 PMID:28362024

Hatton, T. (2013). How have Europeans grown so tall? Oxford Economic Papers, 66(2), 349-372. https://doi.org/10.1093/oep/ gpt030

Hatton, T. J., \& Bray, B. E. (2010). Long run trends in the heights of European men, $19^{\text {th }}-20^{\text {th }}$ centuries. Economics and Human Biology, 8(3), 405-413. https://doi.org/10.1016/j.ehb.2010. 03.001 PMID:20399715

Hermanussen, M., \& Scheffler, C. (2016). Stature signals status: The association of stature, status and perceived dominance - a thought experiment. Anthropologischer Anzeiger, 73(4), 265-274. https:// doi.org/10.1127/anthranz/2016/0698 PMID:27643683

Hermanussen, M., Alt, C., Staub, K., Aßmann, C., \& Groth, D. (2014). The impact of physical connectedness on body height in Swiss conscripts. Anthropologischer Anzeiger, 71(4), 313-327. PMID:25774949

Hermanussen, M., Aßmann, C., Staub, K., \& Groth, D. (2016). Monte Carlo simulation of body height in a spatial network. European Journal of Clinical Nutrition, 70(6), 756. https://doi. org/10.1038/ejcn.2016.71 PMID:27248447

Hijmans, R. (2017). geosphere: Spherical Trigonometry. R package version 1.5-7. https://CRAN.R-project.org/package=geosphere

Hijmans, R., Garcia, N., Wieczorek, J., Rala, A., Maunahan, A., \& Kapoor, J. (2016). GADM database of Global Administrative Areas, Version. Berkeley: University of California. [Online; accessed 04-August-2016], https://www.gadm.org

Holm, S. (1979). A simple sequentially rejective multiple test procedure. Scandinavian Journal of Statistics, 6, 65-70.

Huchard, E., English, S., Bell, M. B., Thavarajah, N., \& CluttonBrock, T. (2016). Competitive growth in a cooperative mammal. Nature, 533(7604), 532-534. https://doi.org/10.1038/nature17986 PMID:27225127

Kobyliansky, E., \& Arensburg, B. (1977). Changes in morphology of human populations due to migration and selection. Annals of Human Biology, 4(1), 57-71. https://doi.org/10.1080/030144677 00001961 PMID:843066

Kolmogorov, A. (1933). Sulla determinazione empirica di una legge di distribuzione. Giornale dell'. Istituto Italiano degli Attuari, 4, 83-91.

Komlos, J. (2009). Anthropometric history: An overview of a quarter century of research. Anthropologischer Anzeiger, 67(4), 341356. https://doi.org/10.1127/0003-5548/2009/0027 PMID: 20440956

Komlos, J. (2010). The recent decline in the height of AfricanAmerican women. Economics and Human Biology, 8(1), 58-66. https://doi.org/10.1016/j.ehb.2009.12.004 PMID:20071251 
Koziel, S., \& Gomula, A. (2017). Variation of height and BMI within school classes in 14-year-old children. Anthropologischer Anzeiger, 74(1), 77-80. https://doi.org/10.1127/anthranz/2017/ 0702 PMID:28362020

Krzyżanowska, M., \& Mascie-Taylor, C. G. (2011). Geographical variation and migration analysis of height, weight and body mass index in a British cohort study. Journal of Biosocial Science, 43(6), 733-749. https://doi.org/10.1017/S0021932 011000381 PMID:21794200

Little, M. A., Gray, S. J., \& Leslie, P. W. (1993). Growth of nomadic and settled Turkana infants of northwest Kenya. American Journal of Physical Anthropology, 92(3), 273-289. https://doi. org/10.1002/ajpa.1330920305 PMID:8291619

Meyer, D., Dimitriadou, E., Hornik, K., Weingessel, A., \& Leisch, F. (2017). e1071: Misc Functions of the Department of Statistics, Probability Theory Group (Formerly: E1071), TU Wien. R package version 1.6-8. https://CRAN.R-project.org/package= e1071

Myburgh, J., Staub, K., Rühli, F. J., Smith, J. R., \& Steyn, M. (2017). Secular trends in stature of late $20^{\text {th }}$ century white South Africans and two European populations. Homo, 68(6), 433-439. https://doi.org/10.1016/j.jchb.2017.10.001 PMID:29079296

NCD Risk Factor Collaboration (2016). A century of trends in adult human height. Elife, 5,e13410.

Pebesma, E., \& Bivand, R. (2005). Classes and methods for spatial data in R. $R$ News, 5(2), 9-13. Retrieved from https:/CRAN.Rproject.org/doc/Rnews/

Pospisil, C., Czernitzki, A.-F., \& Scheffler, C. (2017). No association between nutrition and body height in German kindergarten children - a pilot study. Anthropologischer Anzeiger, 74(3), 199-202. https://doi.org/10.1127/anthranz/2017/0704 PMID: 28555243

R Core Team (2018). R: A Language and Environment for Statistical Computing. Vienna, Austria: R Foundation for Statistical Computing. https://www.R-project.org/

Rietveld, I., Janssen, J. A., van Rossum, E. F., Houwing-Duistermaat, J. J., Rivadeneira, F., Hofman, A., . . Lamberts, S. W. (2004). A polymorphic CA repeat in the IGF-I gene is associated with gender-specific differences in body height, but has no effect on the secular trend in body height. Horumon To Rinsho, 61(2), 195203. https://doi.org/10.1111/j.1365-2265.2004.02078.x PMID: 15272914

Signorell, A., et al. (2018). DescTools: Tools for Descriptive Statistics. R package version 0.99.24. https://cran.r-project.org/ package $=$ DescTools

Silventoinen, K. (2003). Determinants of variation in adult body height. Journal of Biosocial Science, 35(2), 263-285. https:// doi.org/10.1017/S0021932003002633 PMID:12664962

Singh, L. P., \& Harrison, G. A. (1996). Impact of migration, environment and socioeconomic conditions on the physique of Sikhs. Journal of Biosocial Science, 28(1), 101-116. https://doi. org/10.1017/S0021932000022124 PMID:8690738
Smirnov, N. (1939). Estimate of deviation between empirical distribution functions in two independent samples. Bulletin Moscow University, 2, 3-16.

Steckel, R. (1983). Height and per capita income. Historical Methods: A Journal of Quantitative and Interdisciplinary History , 16(1), 1-7. https://doi.org/10.1080/01615440.1983.10 594092

Steckel, R. (2009). Heights and human welfare: Recent developments and new directions. Explorations in Economic History, 46(1), 1-23. https://doi.org/10.1016/j.eeh.2008.12.001

Stulp, G., \& Barrett, L. (2016). Evolutionary perspectives on human height variation. Biological Reviews of the Cambridge Philosophical Society, 91(1), 206-234. https://doi.org/10.1111/ brv.12165 PMID:25530478

Stulp, G., Pollet, T. V., Verhulst, S., \& Buunk, A. P. (2012). A curvilinear effect of height on reproductive success in human males. Behavioral Ecology and Sociobiology, 66(3), 375-384. https:// doi.org/10.1007/s00265-011-1283-2 PMID:22389549

Stulp, G., Buunk, P. A., Verhulst, S., \& Pollet, V. T. (2013). Tall claims? Sense and nonsense about the importance of height of US presidents. The Leadership Quarterly, 24(1), 159-171. https://doi.org/10.1016/j.leaqua.2012.09.002

Stulp, G., Simons, M. J., Grasman, S., \& Pollet, T. V. (2017). Assortative mating for human height: A meta-analysis. American Journal of Human Biology, 29(1), e22917. https://doi.org/ 10.1002/ajhb.22917 PMID:27637175

Watts, D. J., \& Strogatz, S. H. (1998). Collective dynamics of 'small-world' networks. Nature, 393(6684), 440-442. https:// doi.org/10.1038/30918 PMID:9623998

Wei, T., \& Simko, V. (2017). R package corrplot: Visualization of a Correlation Matrix. (Version 0.84). https://github.com/taiyun/ corrplot

Manuscript received: 08 March 2019

Revisions requested: 15 April 2019

Revised version received: 27 May 2019

Accepted: 28 May 2019

\section{The pdf version of this paper includes an electronic supplement}

Please save the electronic supplement contained in this pdf-file by clicking the blue frame above. After saving rename the file extension to .zip (for security reasons Adobe does not allow to embed .exe, .zip, .rar etc. files).
Table of contents - Electronic Supplementary Material (ESM)
ESM 1. Supplementary Table 1. swissdata2015.
ESM 1. Supplementary Table 2. AdjacencyMatrix 